\title{
Micromechanical modelling of nanocrystalline and ultrafine grained metals: A short
} overview

\section{Mishnaevsky, Leon; Levashov, Evgeny}

\section{Published in:}

Computational Materials Science

Link to article, DOI:

10.1016/j.commatsci.2014.09.024

Publication date:

2015

Document Version

Peer reviewed version

Link back to DTU Orbit

Citation (APA):

Mishnaevsky, L., \& Levashov, E. (2015). Micromechanical modelling of nanocrystalline and ultrafine grained metals: A short overview. Computational Materials Science, 96, 365-373.

https://doi.org/10.1016/j.commatsci.2014.09.024

\section{General rights}

Copyright and moral rights for the publications made accessible in the public portal are retained by the authors and/or other copyright owners and it is a condition of accessing publications that users recognise and abide by the legal requirements associated with these rights.

- Users may download and print one copy of any publication from the public portal for the purpose of private study or research.

- You may not further distribute the material or use it for any profit-making activity or commercial gain

- You may freely distribute the URL identifying the publication in the public portal

If you believe that this document breaches copyright please contact us providing details, and we will remove access to the work immediately and investigate your claim 


\title{
MICROMECHANICAL MODELLING OF NANOCRYSTALLINE AND ULTRAFINE GRAINED METALS: A short overview
}

\author{
Leon MISHNAEVSKY Jr ${ }^{1}$ and Evgeny LEVASHOV ${ }^{2}$ \\ ${ }^{1}$ Technical University of Denmark, DTU Wind, Ris $\emptyset$ Campus, \\ DK-4000 Roskilde, Denmark, lemi@dtu.dk \\ ${ }^{2}$ National University of Science and Technology "MISIS", \\ Moscow 119049, Russia
}

\begin{abstract}
:
An overview of micromechanical models of strength and deformation behaviour of nanostructured and ultrafine grained metallic materials is presented. Composite models of nanomaterials, polycrystal plasticity based models, grain boundary sliding, the effect of nonequilibrium grain boundaries and nanoscale properties are discussed and compared. The examples of incorporation of peculiar nanocrystalline effects (like large content of amorphous or semi-amorphous grain boundary phase, partial dislocation GB emission/glide/GB absorption based deformation mechanism, diffusion deformation, etc.) into continuum mechanical approach are given. The possibilities of using micromechanical models to explore the ways of the improving the properties of nanocrystalline materials by modifying their structures (e.g., dispersion strengthening, creating non-equilibrium grain boundaries, varying the grain size distributions and gradients) are discussed.
\end{abstract}

Keywords: Micromechanics; Finite element modelling; Nanomaterials; Nanocrystalline materials

\section{Introduction}

During recent decades, growing interest of scientific community has been attracted to the nanostructured materials. The expectations on nanostructuring as a way to enhance material performances and to improve competing materials properties are very high, and some of them have been delivered, indeed.

The extraordinary properties of nanocrystalline and ultrafine grained metallic materials (i.e., materials with the grain sizes of the order of several to several hundred nanometers) include superductility at room temperature, high hardness and high strength 
to hardness value (which might be $2 . .7$ times higher than in coarse grained materials), lower elastic modulus, negative Hall-Petch slope, enhanced strain rate sensitivity and difference between tensile and compression response [1-5]. The yield strength of nanocrystalline materials can be up to $5 \ldots 10$ times higher than of coarse-grained materials [6]. Other peculiar effect of nanocrystalline materials are the deviation from Hall-Petch relation at ultrafine and nanoscale grain sizes (below $100 \mathrm{~nm}$ ), which goes into negative Hall Petch slope at about $10 \mathrm{~nm}$, as well as asymmetry of tensile and compressive behaviour and enhanced diffusion properties [7].

In order to predict the service properties of the materials and to explore the potential and reserves of their improvement, computational models linking the macroscale (service) properties and nanoscale structures are necessary. While the atomistics/molecular dynamics seem to be most natural approach to simulate the nanoscale effects and behaviour, they are limited in time and size scales [5], and can be hardly linked to real service conditions (long term loading of large size parts). That is why the continuum mechanical/micromechanics methods attracted the interest of specialists in nanomaterials as a tool for the computational analysis of service properties and usability of the materials.

Still, the continuum mechanical/micromechanical models of materials are based on the inherent assumptions about the large scale difference between the structural elements (atoms, dislocations, polymer chains) and the considered volume, as well as underlying physical mechanisms and homogeneity of deformation (role of dislocations, e.g.). Thus, micromechanical modelling of nanomaterials in fact pushes the borders of traditional continuum mechanics seeking to incorporate non-mechanical, physical effects into the purely mechanical concept.

In some cases, coupling methodologies are employed to combine atomistic and continuum models. Among the different approaches which combined continuum mechanics approach with atomistic modelling, one may mention FEAt by Kohlhoff and collaborators [8], Quasicontinuum (QC) method by Tadmor and colleagues [9-11], coarse-grained molecular dynamics (CGMD) by Rudd and Broughton [12], molecularatomistic-ab initio dynamics (MAAD) by Broughton et al. [13], and the bridging scale decomposition (BSD) by Wagner and Liu [14]. Among the challenges of coupling 
approaches, one should mention the problem of linking regions and overlap area as well as fictitious boundary effects, due to inconsistencies in the formulation of the potential energy [15]. The coupled methods make it possible to introduce the physically based materials parameters or laws into micromechanical models.

In this paper, we present an overview of micromechanical models of nanocrystalline and ultrafine grained materials, their mechanical behaviour, deformation and strength. Composite models, crystal plasticity based models, grain boundary sliding, the effect of non-equilibrium grain boundaries, etc. are reviewed. The main constraints and challenges in the considered models are discussed.

\section{Composite models of nanocrystalline metallic materials}

The main structural feature of nanocrystalline and ultrafine grained materials, apart from the small grain sizes, is the high relative volume of grain boundary surface phase. According to Gleiter [1], the differences between the properties of nano - and coarse structured materials are determined by both the low dimensions of grains and the high volume content of the boundary surface phase in nanomaterials. Suryanarayana [16] noted in his review that ,nanocrystalline metals can be considered to consist of two structural components - the numerous small crystallites ... and a network of intercrystalline region“(Figure 1a shows a real microstructure of ultrafine grained aluminium).

The relatively thick "grain boundary phase" layers between nanograins led some researchers to the idea to use "composite model" to simulate the deformation behaviour of nanocrystalline material (e.g., two-phase or even three-phase model of a nanocrystalline material, with grains and boundaries, as phases). The easiest approach of micromechanics, based on the rule-of-mixture, has been transferred to the nanocrystalline materials $[19,20]$.

Phase mixture models. One of the earliest modelling approaches to the analysis of strength of nanomaterials based on the rule-of- mixture was suggested by Carsley et al. [21]. A material is considered as consisting of two phases: squared grains with bulk properties and the boundary phase, which represent a metallic (amorphous) glass 
material. With this model, Carsley and colleagues studied the grain size softening in nanocrystalline nickel, iron and copper, and observed the change in the Hall-Petch slope at small grain sizes.

Kim, Estrin and colleagues [22-25] applied the rule-of-mixture for two-phase composite (crystalline phase and a grain-boundary phase) to study the plastic deformation and grain size dependence of nanocrystalline metals. Kim developed a micromechanical (cubic unit cell) model of a nanostructured material [22] consisting of a crystallite, boundary phase and triple line junctions. The diffusional (Coble creep and Nabarro-Herring creep) deformation mechanisms were taken into account in the model, as well as the noncrystallographic dislocation glide (only for the grain interior). The grain boundary deformation, controlled by diffusional mechanisms is described as viscous Newtonian behaviour. Viscoplastic constitutive laws, including the dislocation density evolution and diffusion creep (boundary diffusion and lattice diffusion mechanisms) were used for the phases. Inverse Hall-Petch behaviour at low strain rates, when both phases deformation is controlled by diffusion mechanisms, was observed. A composite model with cubic unit cells with four sides and 2 horizontal layers of GB and the cube of grain inside was developed by Zhou et al. [26]. The deformation behaviour of grain interior was described by rate independent plasticity with isotropic hardening law. The deformation of grain boundaries was described by Drucker constitutive equation.

Another version of the composite model is based on the presentation of the "grain boundary phase" as sharp grain boundary surrounded by "grain boundary affected zone" (GBAZ) with varied properties (instead of presenting it as isotropic and homogeneous composite matrix). This concept was proposed by Schwaiger et al. [27], who used a model with two-dimensional grains of hexagonal shape separated by the "grainboundary affected zone (GBAZ)" (considered as softer "region adjoining the grain boundaries in $\mathrm{NC}$ (nanocrystalline) metals where the crystalline lattice is elastically strained despite the ostensible absence of any point defects"). Assuming GBAZ thickness to be 7-10 lattice parameters, and linear hardening constitutive behaviour for the grain interior and the GBAZ, Schwaiger and colleagues simulated the strain-rate sensitivity of nanocrystalline nickel. 
On the basis of Schwaiger et al. [27] model, Li and Weng [6] developed a secantviscosity composite model, where the phases follow unified viscoplastic constitutive law, while the yield stress of grain interior depends on the grain size. The model was applied to predict the strain-rate effect and grain-size dependence of a nanocrystalline nickel.

The main achievement of rule-of-mixture models of nanocrystalline materials consists in the introduction of physical, dislocation density or diffusion based constitutive law of grain boundary phases into the model, and combining it with grain size dependent plasticity for grain interior. This allowed to simulate the inverse Hall-Petch effects and to determine the critical grain sizes even using very simple, cubic micromechanical models.

\section{Multi-element composite models and homogenization}

The real structures of nanocrystalline metals are much more complex than the simplistic cubic or hexagonal unit cells, described in the section 2. As different from usual coarse grained materials, such a complexity has a strongest influence also on the mechanisms of deformation and strength of nanocrystalline materials. Such effects as cooperative grain boundary sliding, grain boundary sliding as well as the interplay between deformation mechanisms of smaller (nanoscale) and ultrafine grain scale grains in the same material cannot be taken into account in the framework of simple one-element unit cell models. Several groups have been working to incorporate real, or quasi-real structural features into micromechanical models of nanocrystalline materials.

Quasi-real multi-grain structure models. Some groups of researchers used the quasireal (e.g., Voronoi tessellation based) representations of structures, with some real structure parameters, to represent realistic microstructures of nanometals [28-31] (see an example Figure 1c). So, Mercier et al. [32] considered nanocrystalline material as an aggregate of spherical grains, with random grain sizes, distributed according to lognormal probability distribution. Using the Taylor-Lin deformation analysis approach to include elasticity effects, and dislocation density based constitutive model, the 
authors analysed the transition from a Hall-Petch regime to the inverse Hall-Petch effect.

Fu et al. [33, 34] and Benson et al. [35] proposed a mantle-core model of nanocrystalline materials, in which a monocrystalline core is surrounded by a grainboundary region mantle with a high work hardening rate. They calculated plastic flow as a function of grain size taking into account the dislocation accumulation rates in grainboundary regions and grain interiors. The thickness of the grain boundary zone was assumed to be grain size dependent. The model allowed simulating the decrease of the Hall-Petch slope in the nanocrystalline domain.

Using the Voronoi type composite model, Collini and Bonardi [30] demonstrated that the strength of nanometals depend on the strain variability, which in turn is controlled by the variation of grain sizes. According to [30], contributions of grains of different sizes to deformation are different. They also showed that the GBs carry $10 \%-20 \%$ higher stresses than GI.

Liu and Mishnaevsky Jr. [31] simulated gradient nanocrystalline titanium, with random graded distribution of grain sizes and graded Voronoi tesselation. They demonstrated that more homogeneous structures ensure higher flow stress and also higher damage resistance than highly gradient structures.

The oversimplified cubic or hexagon unit cell models of nanocrystalline materials do not take into account the variability of the grain sizes, orientations of grain boundaries, and other random structure effects. The advantage of the quasi-real, multigrain composite models of nanometals is the possibility to take into account the different deformation mechanisms of grains with different sizes, which have apparently a strong effect on the deformation under the conditions of grain size dependent deformation.

Homogenization, self-consistent and multilevel composite models. A series of homogenization based models of nanocrystalline materials taking into account various deformation mechanisms has been developed by Capolungo, Cherkaoui and their colleagues [33-40]. In [33], the authors employed the homogenization method to evaluate the grain size effect on the deformation of nanocrystalline materials, assuming elastic- perfect plastic behaviour of grain boundaryphase and elastic-viscoplastic behaviour of grain core phase. In [37-39], Capolungo and colleagues used secant self- 
consistent model of composite, with grains (whose deformation is controlled by dislocation glide) and matrix (including both grain boundaries and triple junctions, whose deformation is controlled by grain boundary dislocation emission and penetration. A two-step hierarchical scale transition technique was applied to model the effect of imperfect interfaces in nanocrystalline copper [40], and their effect on the elastoviscoplastic behaviour of the material. The response of the material (considered as three-phase material) is determined by homogenization (Mori-Tanaka scheme) (at lower scale level) and via field translation method and self-consistent procedure of the homogenized inclusion embedded in an equivalent material (at the higher scale level). This model allows simulating both the grain boundary sliding effect and grain boundary dislocation emission.

The generalized elastoplastic self-consistent model developed by Jiang and Weng [41] is based on Christensen and Lo's scheme [42]. For grain interior, the rate-independent crystal plasticity model was used. The pressure-dependent Drucker law was used for grain boundaries. Also, Ramtani et al [43] used the self-consistent scheme by Christensen and Lo model [42], and Drucker constitutive law for grain boundaries.

In order to obtain effective properties of a nanocrystalline material, Voyiadjis and Deliktas [44] employed multilevel Mori-Tanaka averaging scheme. The dislocationbased deformation model was used for grain interior, and dislocation based nonlocal gradient plasticity theory for the grain boundary.

Apparently, the self-consistent and multiscale models allow taking into account the complex structures of nanocrystalline materials in a more rigorous way as compared with idealized cubic or "windows" -type models. Still, some important effects (like variability of grain sizes or irregularities of grain shapes, both critically influencing the deformation mechanisms) can be averaged out in these models, or require some additional ways of dealing with.

Summarizing, one can see that unit cells used have been developed from simplest cubic unit cells [22, 26] and spheres [32], to hexagons, Voronoi tessellation, 3D models [48]. Also, the homogenization methods have been improved, from easiest rule-of-mixture [22], to more complex composite models, like Christensen and Lo self-consistent 
scheme [43], Mori-Tanaka and hierarchical models [33-40] or real structure models with experimentally determined lognormal grain size distribution [28, 29].

Apart from the large volume content of grain boundaries, the effect of triple joints and peculiarities of the grain boundary phases, plastic anisotropy of grains and the size effects in grains (also, interaction between statistically stored and geometrically necessary dislocations) play an important role for the mechanical behaviour of the nanocrystalline materials. They can be modelled with the use of crystal plasticity approach [49-51], enhanced to include the size effects.

\section{Dislocation mechanisms of deformation and polycrystal plasticity models}

Constitutive laws of phases and special deformation mechanisms. Apparently, the main challenge of all the composite models of nanocrystalline metals is to determine the correct constitutive laws for the grains interior (GI) and grain boundary (GB) phases (which is in fact not a solid body but rather amorphous or partially amorphous materials, with graded properties and structures), reflecting their real properties and deformation mechanisms. In these materials, different deformation mechanisms are active (as different from coarse grained metals where plastic accommodation is controlled by the activity of dislocation sources and conventional dislocation glide). As discussed above, these mechanisms include diffusion controlled deformation (for very small grains), sliding and rotation of grains, as well as the different conditions of emission, glide and absorption of dislocation nucleation or partial dislocations (sometimes accompanied by twinning) [45, 46]. Many of these mechanisms can be active in parallel, interact and interplay and transfer from one to another under some conditions. In order to include such interactions into computational micromechanical models of deformation, corresponding analytical models should be available.

In the models, listed above, the constitutive behaviour of phases is described by elastoplastic or viscoplastic constitutive laws, with hardening, or, at the physical level, by dislocation or diffusion based models. Among the constitutive laws used in some models, one can list the grain size dependent plasticity (for grain interior/GI) and 
amorphous glass model (GB) [21], anisotropic elasto-plastic models with different orientations (GI) and Voce-hardening law with high work hardening rate. Depending on the distance from the closest grain boundary (GB) [33, 35], isotropic, linear hardening behaviour (GI) and isotropic power-law type rate dependent constitutive response (GB) [27], dislocation glide model (GI) and diffusional (Coble creep and Nabarro-Herring creep) deformation [22-25], unified viscoplastic constitutive law[6], elastic-viscoplastic behaviour and dislocation glide (GI) and elastic perfect plastic behaviour incorporating the model of grain boundary dislocation emission and penetration (GB) [33-40], crystal viscoplasticity with Hall Petch grain size dependence (GI) and isotropic viscoplasticity and Mohr-Coulomb pressure dependence (GB) [47] .

Size dependent polycrystal plasticity. The misorientation of nanograins and the availability of high/low angle grain boundaries have a strong effect on the material properties, and can be studied with the use of the crystal plasticity (CP) approach. The scale dependent versions of polycrystals plasticity models, which can be generalized to the nanomaterials, have been realized by incorporating dislocation-density-based constitutive equations $[52,53]$ and the strain gradient crystal plasticity (SGCP) model in the continuum crystal plasticity approach [52-59].

The model of deformation mechanisms in nanocrystalline metals, describing the emission of perfect and partial dislocations, deformation twins from grain boundaries, and grain boundary sliding was developed by Asaro, Krysl, and Kad [62] (thus, called AKK model). Using this approach, Zhu, Asaro and colleagues [60, 61] developed a polycrystalline constitutive model of nanocrystalline materials (electrodeposited $\mathrm{Ni}$ ), and studied the effect of the grain size distribution (taken as lognormal probability distribution) on the mechanical behaviour of nanonickel. The model takes into account the simultaneous contributions of deformation mechanisms including grain boundary emission of dislocations and/or stacking faults, as well as for grain boundary sliding.

Wei and Gao [63] extended this model by Zhu et al. [60] by including the deformation by grain boundary diffusion. Using the developed elastic-viscoplastic model, which incorporates all the main deformation mechanisms, grain-boundary sliding, grainboundary diffusion, grain-interior diffusion and grain-interior plasticity, they studied the 
deformation of $\mathrm{nCu}$ and showed that the relative contribution of different mechanisms depends on both grain size and the applied strain rate.

Using crystal plasticity approach either in both phases or only in grain interior (with GBs described by perfectly plastic isotropic Voce equation) Fu et al. [33] studied the grain size effects and deformation mechanisms. The grain boundary was assigned lower yield stress and higher work hardening rate than grains. Further, the authors modelled the material behaviour with a hardening model related to the dislocation density evolution. The authors observed accelerated hardening in the grain-boundary regions, as well as shear localization in their simulations.

Assuming that the plastic deformation is controlled by dislocations which nucleate at a GB, travel through the grain, and are absorbed by the next GB, Warner and Molinari [65] developed a semi-discrete and non-local crystal plasticity model. Nor dislocationdislocation interactions neither partial dislocations are included. They observed larger stress heterogeneities and intragranular plasticity than could be observed with traditional crystal plasticity models.

A micromechanical model developed by Lebensohn et al. [47] is based on fast Fourier transforms. Unit cells with self-similar polycrystals of different grain sizes were designed, using the crystal viscoplasticity with Hall-Petch grain size dependence for the grain interior and isotropic viscoplasticity for the grain boundaries. The authors studied the effect of grain size, strain rate and pressure on the local and overall behaviour of ncmaterials under quasi-static and shock loading conditions.

Gürses and El Sayed [66,67] developed a variational two-phase constitutive model, with rate-independent anisotropic crystal plasticity model for grain interior (taking into account the transition from the partial to full dislocation based plasticity) and ratedependent isotropic porous plasticity model for GBAZ (including the availability of voids and void growth in GBAZ). Using the Taylor-type homogenization, the authors modelled the observed grain size dependent rate-sensitivity of the behaviour of nanocrystalline FCC metals $(\mathrm{nCu}, \mathrm{nNi})$ under finite deformations.

A crystal plasticity model by Aoyagi and colleagues [68] incorporates the effects of grain boundaries and dislocation sources (included through hardening law of crystal 
plasticity). The authors investigated the grain size effect on the yield stress of in UFG SPD produced materials.

The dislocation-density grain boundary interaction (DDGBI) model between GBs and adjacent grain interiors, developed by Shi and Zikry [69], includes dislocation-density absorption, emission and transmission and allows modelling the grain size effect. The constitutive formulation for the finite deformation of rate dependent multiple-slip crystal plasticity is used. The GB misorientation dependent dislocation-density relation is taken into account. The yield stress as a function of the grain size was obtained.

Li et al. [70] implemented quantized crystal plasticity (QCP) into FE model of 1000 grain polycrystals. Based on the results of MD simulations, the authors studied the plastic deformation and strain evolution and localization in nanometals, and observed the grain size dependent jumps in the plastic strains.

Segurado and LLorca [71] developed the 3D crystal plasticity (CP) model of nanocrystalline titanium taking into account the real grain orientation distribution. They used two different representations of the microstructure were used: a voxel-based model from cubic finite elements (each of them representing a Ti grain), and a model where each crystal is represented with many elements. In either RVE of the polycrystal, the orientation of each grain was determined from the input orientation distribution function (ODF) which describes the initial texture using a Monte Carlo method.

Size dependent polycrystal plasticity models, incorporating real deformation mechanisms of nanocrystalline metals (partials, diffusion deformation, other effects and interplay between them) make it possible to reflect both polycrystalline structure and nanocrystalline effects in the materials deformation.

\section{Grain boundary sliding}

The grain boundary sliding and grain rotation are important mechanisms of deformation of nanocrystalline and ultrafine grained materials. It is quite often assumed that the grain boundary sliding is co-responsible for the superductility of nanocrystalline materials [72]. The GBS in nanocrystalline materials can be accommodated by dislocation (e.g., emission of lattice dislocations from triple junctions into GI, glide and absorption at 
GBs [74]), or diffusion mechanisms (e.g., GB diffusion in the vicinity of triple junctions) [75-77], but also by rotational deformation [78]. The grain boundary sliding which accommodates the local strains (so called Rachinger grain boundary sliding) does not increase the number of grains along the length of the specimen, while the GBS accommodating the diffusion (so called Lifshitz grain boundary sliding) leads to the increase of grain number.

Plastic deformation with crystal lattice rotation inside grains is more active at later stages of GBS, when many GBS processes occur in many grain boundaries. The cooperative grain boundary sliding (CGBS) occurs as sliding and rotation of groups of grains [79], and is one of the mechanisms for superplasticity in nanocrystalline materials.

Ashby and Verrall described and modeled a mechanism of deformation of polycrystalline materials when "grains switch their neighbors and do not elongate significantly" [73]. They presented a "four grain model" of grains switching neighbors and derived the constitutive law for diffusion accommodated flow.

Raj and Ashby [72] considered the problem of sliding of nonplanar periodically waved or stepped grain boundary analytically, and obtained interrelationships between the sliding rate, rate of diffusion creep and the grain boundary shapes. Their equations have been used in a number of following works. Figure 2 shows a schema of GBS path accommodated by diffusion flow, as assumed in [33, 72].

Representing grains as rhombic dodecahedrons and considering rate controlled flow of grain boundaries, Hahn and Padmanabhan $[80,81]$ derived an equation for the threshold stress needed for mesoscopic grain boundary sliding and studied the inverse Hall-Petch effect in nanomaterials.

A number of computational models of the nanomaterial deformation are based on the analytical models from $[72,73,80]$.

In their elastic-viscoplastic model of deformation, which takes into account the competition among various deformation mechanisms, Wei and Gao [63] considered GBS as consisting of thermally activated sliding and rate-independent athermal components (relative sliding of two grain boundaries when the resolved shear stress 
overcomes a threshold resistance), and employed the Conrad-Narayan model [64] to determine the macroscopic shearing rate of GBS. They determined fractions of plastic strain due to different mechanisms. Also, Zhu et al. [60] modelled grain boundary sliding using the phenomenological kinetic relations by Conrad and Narayan and analyzed the transition between dislocations based deformation and GBS. They concluded that GBS starts to contribute to the deformation at the grain size below 15 $\mathrm{nm}$.

Shi and Zikry [69] incorporated the Raj-Ashby model of GBS in their dislocationdensity grain boundary interaction (DDGBI) based model, and simulated the GBS displacement for random low angle aggregates with various grain sizes. They observed that GBS increases when grain size decreases, and that GBS can change the direction of crack growth or even cause intergranular crack.

Yang and Wang [82] developed of a constitutive model of cooperative GBS (in 9-grain cluster) based on Ashby and Verall approach [73]. They considered nanograins in 9grain cluster sliding pass one another, taking into account two mechanisms (rotation of grain pairs and varying inter-grain distance) considered as cooperative deformation mechanisms. They observed linear relation between applied stress and creep rate in their simulations.

Ovid'ko and Sheinerman [78] modelled the grain boundary sliding accommodated by the rotational deformation of nanocrystalline nickel, which is in turn realized visa diffusion controlled climb of GB dislocations as well as GBS, using the dislocation dynamics approach incorporates dislocation climb over GB. They observed strong strain hardening at the initial stages of deformation, but constant flaw stress at later stages of deformation.

With view on the possibility of additional diffusion routes in triple junctions contributing to the accommodation of GB sliding, Rabkin and colleagues [2] derived equations for the strain rate of nanocrystalline material due to GB sliding which take into account the triple junction diffusion controlled sliding. They have shown that dependence of deformation rate on the grain size is stronger in the case of triple junction diffusion controlled sliding than in the case when GB sliding is controlled by the GB diffusion. They also investigated the GB dislocation nucleation (e.g., partial dislocation) 
accompanied by atomic shuffles and stress-assisted free volume migration at the GB using the developed "toy model" of the GB sliding.

In several works, variational and kinematic approaches and cohesive/interface finite elements were employed to analyse the grain boundary sliding in nanocrystalline materials. So, Voyiadjis and Deliktas [44] characterized the grain boundary sliding and separation by energetic and dissipative state variables (taking into account internal contact surfaces) in their multilevel Mori-Tanaka/strain gradient plasticity model.

A 3D continuum model of nanometals with tetrakaidecahedra-shaped grains and grain boundaries presented as discontinuity layers of finite thickness was developed by Jerusalem et al. [48]. Grain-boundary sliding and accommodation are described with the use of variational constitutive laws, and simulated with the use of interface elements. The model allowed simulating the reverse Hall-Petch size relationship in nanocrystalline copper. In [83], Jerusalem and colleagues developed a phenomenological model of grain boundary sliding including Mohr-Coulomb friction reflecting the decrease of sliding resistance at higher applied stress.

Warner and colleagues [84] studied the grain boundary sliding using FE model based on atomistic analysis (quasicontinuum method). The grain interior was modelled using the classical rate dependent CP model, while the GB cohesive law parameters were obtained from atomistic simulations. The Voronoi tessellation based model with lognormal distribution of grain sizes was used. The authors investigated the interaction between GB sliding and intragranular plasticity,

In their homogenization model, Barai and Weng [85] incorporated the grain-boundary sliding at the interface between the grain and GB zone as the Newtonian flow. Studying the effect of interfacial sliding viscosity on the material strength, they observed a transition from the low to higher (saturation) strength with increasing the sliding viscosity.

Grain boundary sliding represents a large deformation process, interacting with and being influenced by atomistic processes, like diffusion and dislocation evolution. Thus, the directions of modelling GBS range from the variational continuum and phenomenological models, on the one side, to the atomistic based physical models, on 
the other side. The promising directions of modelling lie in combining continuum and physical models of the process.

\section{Non-equilibrium grain boundaries and GB defects in nanocrystalline materials}

The potential of grain boundaries as a source of the material improvement has been demonstrated in a number of works, e.g. on "grain boundary engineering" [86, 87], on dispersion strengthening of nanomaterials [88] and also discussed in previous sections. The viscosity, deformation behavior, dislocation density, deformation mechanisms, shapes and structures of grain boundaries determine the strength and deformation of nanocrystalline materials.

Non-equilibrium grain boundaries. One of the interesting solutions of the materials property enhancement lies in the concept of non-equilibrium grain boundaries (NEGB) [87]. Recently, several works appeared which show that the mechanical properties of nanomaterials can be potentially improved by using non-equilibrium state of the grain boundaries [87, 90-91].

Typical features of non-equilibrium grain boundaries are the higher energies in GBs, large amount of GB dislocations, higher diffusion coefficient, larger free volume, concentration of alloying elements and formation of precipitates near boundaries [37, 92-96]. However, there are still different views and approaches both on how to characterize the degree of non-equilibrium in grain boundaries and also on the effect of the non-equilibrium state on the mechanical properties. Apart from indirect experiments, atomistic simulations have been used to clarify the role of "nonequilibrium" state for the materials properties. So, Tucker and McDowell [93] used the excess GB free volume as a measure of the degree of 'non-equilibrium' state of grain boundaries. On the basis of atomistic simulations, they have shown that for NEGBs (non-equilibrium grain boundaries), the peak shear stress is lower than that of the corresponding EGBs (equilibrium grain boundaries). Under uniaxial tension, the peak stress (tensile strength) is lowered as a function of increasing NE state. Under shear, resistances to GB sliding and migration decrease with increasing the excess interfacial free volume. Hasnaoui et al. [94] concluded that annealed samples (with ordered, 
equilibrium GBs) impede GBS and show reduced plastic strain and increased strength. In disordered GB, the increase in plastic strain occurs at earlier stages of deformation, but later the strain rates converge for EQ and NE samples (roughly, 40\% increase in strain).

Due to the relative novelty of the concept, and limited information of the acting physical mechanisms, only a few micromechanical studies on non-equilibrium grain boundaries have been carried out. So, Liu et al. [31, 97] studied the influence of NEGB on materials deformation, including varied diffusion coefficient and high initial dislocation density in GB with their micromechanical models. They used a hexagonal and Voronoi-based composite model, and the ABAQUS subroutine VUMAT based on the dislocation density evolution model of GB deformation. It was demonstrated that the nonequilibrium of GBs leads to the increase in the yield stress with decreasing the diffusion coefficient and increasing the initial dislocation density (DD).

Péron-Lührs et al. [98] developed a multiscale model to analyze the effect of the grain boundary character distribution (i.e., high angle and low angle boundaries distribution) on the mechanical behavior. The mechanical responses of the grain boundary (including sliding and decohesion) and grain were obtained using quasicontinuum method (QC), and introduced into the continuum FE model. The two scale model takes into account the grain GB network crystallographic orientation.

While the formation of nanocrystalline materials with disordered, non-equilibrium grain boundaries has a potential for the improvement of material properties, further investigations view on the non-equilibrium effects and their mechanisms are required, to provide input data for the reliable modelling of NEGB effects.

Secondary phases, foreign inclusions and precipitates in grain boundaries. The effect of non-equilibrium grain boundaries is also related to the concentration of alloying elements and formation of precipitates and segregations near grain boundaries, which might increase the critical stresses necessary for nucleation of new dislocations at the boundaries and/or for their motion, and have also a potential for the property enhancement $[88,89]$. 
In their classical paper [72], Raj and Ashby discussed the effect of GB precipitations on the deformation and GBS, and noticed that the precipitates can restrict the grain boundary sliding, by influencing the diffusional creep and the ability of GBs to absorb/emit vacancies.

Using the approach developed in [29] (multigrain Voronoi based composite model), Dobosz et al. [99] studied numerically the effect of second phase particles at grain boundaries. They concluded that the particles located at GBS may lead to the material strengthening, if the material is deformed by GBS mechanism; otherwise, their effect is negligible. They lead also to the higher work hardening rate.

Liu et al. [97] and Liu and Mishnaevsky Jr. [31] studied the influence of foreign inclusions (which might represent physically dispersoids or precipitates caused by foreign atoms) on the mechanical behavior and damage initiation in ultrafine grained (UFG) titanium numerically, using their Voronoi-based model. They compared precipitates located randomly in the grain boundary (GB) layer, on the "interface" between grain interior (GI) and GB and inside grains. It was observed that precipitation has quite a significant effect on the mechanical behavior of UFG Ti. Precipitates/foreign atoms (e.g., oxygen and carbon precipitates) in the GB lead to the increased yield stress, with roughly 2 times stronger effect of precipitations on GB/GI border than randomly arranged in GB. Precipitates located on the GB/GI interface lead to a higher mechanical strength than precipitates located randomly inside the GBs.

Precipitates and dispersions located in grain boundaries but also in grains of nanocrystalline materials represent a promising way to enhance and control the strength and mechanical properties of the materials. The computational studies of the dispersion and precipitate effects listed above are based on the assumption about dislocation mechanisms of precipitate-metal interaction. In order to explore the potential of precipitates for nanomaterials with diffusion based or other deformation mechanisms, additional studies of precipitate-diffusion interaction might be needed.

Defect role and defect evolution in grain boundary phases. The structure of grain boundaries evolves during the deformation, among others, by formation of voids and microcracks. Given the lack of clarity about mechanisms of deformation of 
nanomaterials and complex interplay of many interacting mechanisms, the modelling of damage and degradation of nanomaterials remains still a challenge.

Still, there are a number of investigations of damage mechanisms in nanocrystalline materials [5]. Meyers et al. [5] noticed that the damage initiates "at discrete sites, that are, most often, triple junctions and grain boundaries". Analyzing the void initiation in nanonickel, Kumar et al. [100, 101] listed the following mechanisms: existing voids at grain boundaries and triple points, voids created by dislocation emission from grain boundaries, voids created by grain-boundary sliding which leaves wedges at triple junction, cavities created by the action of grain-boundary sliding on the ledges. Cizek et al. [102] observed microvoids (formed as clusters of vacancies on the grain boundaries created during SPD) in different UFG metals. The fracture surfaces in UFG and nanostructured metals are characterized by dimples which can be however larger than the grain sizes [103]. Chuvil'deev [104] wrote that the damage in nanomaterials is due to the disclination type defect formation at triple grain junctions (these defects can be presented as biaxial disclination dipoles).

In order to simulate the damage initiation and evolution in nanocrystalline and UFG materials, a number of models based on cohesive interface approach [105-107], void growth analysis [109] and various damage criteria [27, 31] have been developed.

Wei and Anand [105] developed a composite model with single-crystal plasticity constitutive model for the grain interior, and cohesive interface model for grain boundary (accounting for elastic deformation and irreversible sliding and separation). Further, using the new strain-rate-dependent continuum-plasticity theory for amorphous metals, Wei and colleagues [106] developed a modified version of the model of the deformation and failure of NC FCC metals, describing the grain boundary behavior with this theory. They used a rate-dependent amorphous plasticity model incorporating cavitation and damage for the grain boundaries phase, and $\mathrm{CP}$ approach for the grain interior in their FE model of $\mathrm{nNi}$. In the grain interiors the slip system resistances were assumed to be inversely proportional to the grain size. They observed the transition of deformation mechanism from intragranular shearing to GB shears when the grain size approaches $10 \mathrm{~nm}$. They also observed the cavitation at triple-junctions as a result of grain-boundary shearing in the simulations. 
Chen and Wei [107] employed the mixed-mode cohesive interface model to simulate the grain boundary damage evolutions. The strain gradient plasticity theory is used for grain interior. The authors simulated the overall mechanical response and damage evolution in $\mathrm{nCu}$ and $\mathrm{nNi}$, and confirmed the decisive role of $\mathrm{GB}$ strength and damage behavior for overall material response.

To analyse the void growth and coalescence in the grain boundaries, Siddiq and Sayed [109] proposed a variational multiscale constitutive model of nanocrystalline material, represented as grain/GBAZ composite. The model includes the anisotropic crystal plasticity approach (taking into account partial dislocations) for GI, and isotropic rateindependent porous plasticity model with deviatoric and volumetric plasticity as well as void coalescence for GBAZ.

In their GBAZ composite model, Schwaiger et al. [27], postulated a strain-based material damage criterion and employed it to model onset and progression of failure in nanonickel. Liu and Mishnaevsky Jr. [31] employed Voronoi based composite models and dislocation density based constitutive laws in grain interior and grain boundary phases, and inverse modelling, to determine the most appropriate damage initiation criteria in grain boundaries. Comparing several damage criteria for ductile materials (critical plastic strain, Rice-Tracey void formation, etc. [110, 111]), the demonstrated the dislocation based creep damage model developed by Li and colleagues [112, 113] allows describing the damage initiation in nanocrystalline titanium well. Figure 3 shows the damage distribution in the multigrain model of ultrafine grained titanium with precipitates in grains and grain boundaries, obtained using this model [31].

\section{Conclusions}

In this paper, a short overview of micromechanical models of strength and deformation of nanocrystalline and ultrafine grained metallic materials is given. The methods based on composite/phase mixture models, quasi-real Voronoi based, self-consistent and multiscale composite models of nanomaterials, polycrystals plasticity models, as well as micromechanical models of grain boundary sliding, non-equilibrium grain boundaries and damage initiation in nanomaterials are reviewed. 
In the framework of micromechanics, peculiarities of nanomaterials structures and deformation (large content of amorphous or semi-amorphous grain boundary phase, partial dislocation GB emission/glide/GB absorption based deformation mechanism, diffusion deformation, no dislocations in grain interior, and size dependent interplay of all these mechanisms, combined crystal plasticity/dislocation/grain boundaries effects) can be incorporated into continuum mechanical approach. Using the models, parameter studies can be carried out, to clarify various effects controlling the strength of the materials.

The main challenge of the computational modeling of nanocrystalline and ultrafine grained materials is the limited knowledge about the real mechanisms of deformation and strength for given materials and loading conditions. The information about the deformation mechanisms comes in most cases from indirect experiments, inverse analysis (formulation of model, fitting its parameters, comparing to experiments), atomistic/MD simulations, and seldom from direct experiments.

Micromechanical models make it possible to explore the potential of the properties enhancement of nanocrystalline and UFG materials by varying their structures, e.g. in the framework of numerical experiments. In micromechanical studies, it was shown that dispersion strengthening, creating non-equilibrium grain boundaries, varying the grain size distributions and gradients, controlling the deformation mechanisms can be exploited to improve the properties of nanocrystalline materials.

Acknowledgement: L.M. gratefully acknowledges the financial support of the Danish Council for Strategic Research (DSF) via the Sino-Danish collaborative project "High reliability of large wind turbines via computational micromechanics based enhancement of materials performances" (Ref. no. 10-094539). The authors acknowledge also the financial support of the Ministry of Education and Science of the Russian Federation in the framework of Increase Competitiveness Program of NSTU MISIS. The authors are grateful to Professor Niels Hansen (DTU) and Professor Ruslan Valiev (Ufa State Aviation Technical University) for important discussions and advices.

\section{References:}

1. H. Gleiter, Nanostructured materials: basic concepts and microstructurep Acta Mater.48 (2000) 1. 
2. L. Mishnaevsky Jr., E. Levashov, R. Valiev, E.Rabkin, E. Gutmanas et al. Nanostructured titanium based materials for medical implants: Modeling and development, Materials Science and Engineering R: Reports. Vol. 81, 2014, pp. 1-19

3. C. Suryanarayana and C.C. Koch, Nanocrystalline materials - Current research and future directions, Hyperfine Interactions 130:5-44, 2000

4. S. Divinski, H. Rösner, G. Wilde, Functional Nanostructured Materials - Microstructure, Thermodynamic Stability and Atomic Mobility, Chapter 1, Frontiers of Nanoscience, Volume 1, 2009, Pages 1-50

5. M.A. Meyers, A. Mishra, D.J. Benson, Mechanical properties of nanocrystalline materials, Progress in Materials Science, Volume 51, Issue 4, May 2006, Pages 427-556

6. J. Li, G.J. Weng, A secant-viscosity composite model for the strain-rate sensitivity of nanocrystalline materials, International Journal of Plasticity, Volume 23, Issue 12, 2007, Pages 2115-2133

7. Y. Wei, A.F. Bower, H.J. Gao, Enhanced strain-rate sensitivity in fcc nanocrystals due to grain-boundary diffusion and sliding, Acta Materialia, Volume 56, Issue 8, May 2008, Pages 1741-1752

8. S. Kohlhoff, P. Gumbsch and H.F. Fischmeister, Crack propagation in BCC crystals studied with a combined finite-element and atomistic model, Philosophical Magazine A 64 (1991), pp. 851-878.

9. E.B. Tadmor, M. Ortiz and R. Phillips, Quasicontinuum analysis of defects in solids, Philosophical Magazine A 73 (1996) (6), pp. 1529-1563

10. V.B. Shenoy, R. Miller, E.b. Tadmor, D. Rodney, R. Phillips, M. Ortiz, An adaptive finite element approach to atomic-scale mechanics - the quasicontinuum method, Journal of the Mechanics and Physics of Solids, Volume 47, Issue 3, 1 March 1999, Pages 611-642

11. R. Miller, M. Ortiz, R. Phillips, V. Shenoy, E.B. Tadmor Quasicontinuum models of fracture and plasticity, Engineering Fracture Mechanics, Volume 61, Issues 3-4, September-November 1998, Pages 427-444

12. E. Rudd and J.Q. Broughton, Coarse-grained molecular dynamics and the atomic limit of finite elements, Physical Review B 58 (1998), pp. R5893-R5896

13. Q. Broughton, F.F. Abraham, N. Bernstein and E. Kaxiras, Concurrent coupling of length scales: methodology and application, Physical Review B 60 (1999), pp. 2391-2403

14. G.J. Wagner and W.K. Liu, Coupling of atomistic and continuum simulations using a bridging scale decomposition, Journal of Computational Physics 190 (2003), pp. 249-274

15. P.A. Klein, J.A. Zimmerman Coupled atomistic-continuum simulations using arbitrary overlapping domains, J Computational Physics, Vol. 213, No 1, 2006, pp. 86-116

16. C. Suryanarayana, (1995) Nanocrystalline Materials. International Materials Reviews, 40 (2), pp. 41- 63

17. O.V. Mishin, V.M. Segal and S. Ferrasse Quantitative microstructural characterization of thick aluminum plates heavily deformed using equal channel angular extrusion, , Metallurgical and Materials Transactions A, December 2012, Volume 43, Issue 12, pp 47674776

18. J. Hagstroem, O.V. Mishin, and B. Hutchinson: Gallium enhanced microscopy for revealing grain boundaries and dislocation subboundaries in aluminium alloysScripta Mater., 2003, vol. 49, pp. 1035-40

19. L. Mishnaevsky Jr, Computational Mesomechanics of Composites, John Wiley, 2007, 280 pp. 
20. L. Mishnaevsky Jr., S. Schmauder, 2001, Continuum mesomechanical finite element modeling in materials development: a state-of-the-art review, Applied Mechanics Reviews, $54,1,49-69$

21. Carsley, J.E., Ning, J., Milligan, W.W., Hackney, S.A. and Aifantis, E.C. (1995) A simple, mixtures-based model for the grain size dependence of strength in nanophase metals, Nanostructured Materials, 5 (4), pp. 441-8

22. Kim, H.S., (1998) A composite model for mechanical properties of nanocrystalline materials, Scripta Mater. 39 (8) pp. 1057-1061

23. Kim, HS; Estrin, Y; Bush, MB Plastic deformation behaviour of fine-grained materials, Acta Materialia Volume: 48 Issue: 2 Pages: 493-504, 2000

24. Kim, HS; Estrin, Y, Phase mixture modeling of the strain rate dependent mechanical behavior of nanostructured materials, acta materialia Volume: 53 Issue: 3 Pages: 765-772 Published: FEB 2005

25. Kim, HS; Estrin, Y; Bush, MB, Constitutive modelling of strength and plasticity of nanocrystalline metallic materials, Materials Science And Engineering A Volume: 316 Issue: 1-2 Pages: 195-199 Published: OCT 152001

26. Zhou et al, A mixtures-based model for the grain size dependent mechanical behavior of nanocrystalline materials, Materials Processing Technology, 197 (2008) 325-336

27. R Schwaiger, B Moser, M Dao, N Chollacoop, S Suresh, Some critical experiments on the strain-rate sensitivity of nanocrystalline nickel, Acta Materialia, Volume 51, Issue 17, 6 October 2003, Pages 5159-5172

28. S. Li, J. Zhou, L. Ma, N. Xu, R.T. Zhu, X.H. He Continuum level simulation on the deformation behavior of nanocrystalline nickel, Computational Materials Science, Volume 45, Issue 2, April 2009, Pages 390-397

29. R. Dobosz, M. Lewandowska, K.f J. Kurzydlowski The effect of grain size diversity on the flow stress of nanocrystalline metals by finite-element modelling, Scripta Materialia, Volume 67, Issue 4, August 2012, Pages 408-411

30. L.Collini and A.Bonardi, A micromechanical model of the evolution of stress and strain fields in ultrafine-grained metal structures under tension J Strain Analysis, DOI: 10.1177/0309324712466550, sdj.sagepub.com

31. H.S. Liu, L. Mishnaevsky Jr., Gradient ultrafine-grained titanium: computational study of mechanical and damage behavior, Acta materialia, Vol. 71, 2014, pp. 220-233

32. S. Mercier, A. Molinari, Y. Estrin, Grain size dependence of strength of nanocrystalline materials as exemplified by copper: an elastic-viscoplastic modelling approach, J. Mater. Sci,2007, vol. 42, pp. 1455-1465

33. H.-H. Fu, D.J. Benson, M.A. Meyers, Analytical and computational description of effect of grain size on yield stress of metals, Acta Mater, 49 (2001), p. 2567

34. H.H. Fu, D.J. Benson, M.A. Meyers, Computational description of nanocrystalline deformation based on crystal plasticity, Acta Materialia, 52 (2004), pp. 4413-4425

35. D.J. Benson, H.H. Fu, M.A. Meyers, On the effect of grain size on yield stress: extension into nanocrystalline domain, Mater. Sci. Eng. A, 319-321 (2001), pp. 854-861

36. L. Capolungo, C. Jochum, M. Cherkaoui, J. Qu, Homogenization method for strength and inelastic behavior of nanocrystalline materials, International Journal of Plasticity, Volume 21, Issue 1, January 2005, Pages 67-82

37. L. Capolungo, M. Cherkaoui, J. Qu, A self-consistent model for the inelastic deformation of nanocrystalline materials, Journal of Engineering Materials and Technology_-Transactions of the ASME, 127 (2005), pp. 400-407 
38. L. Capolungo, M. Cherkaoui, J. Qu, On the elastic-viscoplastic behavior of nanocrystalline materials , International Journal of Plasticity, Volume 23, Issue 4, April 2007, Pages 561591

39. S Benkassem,.; L.; Capolungo, M.Cherkaoui, Mechanical properties and multi-scale modeling of nanocrystalline materials, Acta Materialia, Volume: 55 Issue: 10 Pages: 3563-3572 Published: JUN 2007

40. L. Capolungo, S. Benkassem, M. Cherkaoui, J. Qu, Self-consistent scale transition with imperfect interfaces: Application to nanocrystalline materials , Acta Materialia, Volume 56, Issue 7, April 2008, Pages 1546-1554

41. B. Jiang, G.J. Weng, A generalized self-consistent polycrystal model for the yield strength of nanocrystalline materials, J Mech Phys Solids, 52 (2004), p. 1125

42. Christensen, R.M. and Lo, K.H. (1979) Solution for effective shear properties in three-phase sphere and cylinder models, Journal of the Mechanics and Physics of Solids, 27, pp. 315330

43. S. Ramtani, G. Dirras, H.Q. BuiA bimodal bulk ultra-fine-grained nickel: Experimental and micromechanical investigations, Mechanics of Materials, Volume 42, Issue 5, May 2010, Pages 522-536

44. G.Z. Voyiadjis, B. Deliktas, Modeling of strengthening and softening in inelastic nanocrystalline materials with reference to the triple junction and grain boundaries using strain gradient, plasticity, Acta Mech 213, 3-26 (2010

45. H. Van Swygenhoven, M. Spaczer, A. Caro, D. Farkas, Competing plastic deformation mechanisms in nanophase metal. Phys Rev B 60:22Phys. Rev. B. 60 (1999) 22-25

46. M. Zehetbauer, Cold work hardening in stages IV and V of F.C.C. metals-II. Model fits and physical results, Acta Metallurgica et Materialia, Volume 41, Issue 2, February 1993, Pages 589-599

47. R.A. Lebensohn, E.M. Bringa, A. Caro, A viscoplastic micromechanical model for the yield strength of nanocrystalline materials, Acta Materialia, Volume 55, Issue 1, 2007, Pages 261271

48. A. Jérusalem, L. Stainier, R. Radovitzky, $\square$ A continuum model describing the reverse grain-size dependence of the strength of nanocrystalline metals, $\square$ Philos. Mag., 87 (2007), pp. 2541-2559

49. R. Asaro, Micromechanics of Crystals and Polycrystals, in: Advances in Applied Mechanics, Vol. 23, Academic Press, New York, 1983.

50. F. Roters, P. Eisenlohr, L. Hantcherli, D.D. Tjahjanto, T.R. Bieler, D. Raabe, Overview of constitutive laws, kinematics, homogenization and multiscale methods in crystal plasticity finite-element modeling: Theory, experiments, applications , Mater. 58 (2010) 1152-1211.

51. F.Roters, Ph. Eisenlohr, Th.R. Bieler and D. Raabe, Crystal Plasticity Finite Element Methods: in Materials Science and Engineering, Wiley-ICH, 170 pp. 2010

52. H. Lim, M.G. Lee, J.H. Kim, B.L. Adams, R.H. Wagoner, Simulation of polycrystal deformation with grain and grain boundary effects, Int. J. Plasticity, 27 (2011), pp. 13281354

53. T. Ohashi, M. Kawamukai, H. Zbib, A multiscale approach for modeling scale-dependent yield stress in polycrystalline metals, Int. J. Plasticity, 23 (2007), pp. 897-914

54. E.C. Aifantis, On the Microstructural Origin of Certain Inelastic ModelsJ. Eng. Mater. Tech. 106 (1984) 326-330.

55. N.A. Fleck, J.W. Hutchinson, Strain gradient plasticity, Adv. Appl. Mech. 33 (1997) 295361. 
56. N.A. Fleck, J.W. Hutchinson, A reformulation of strain gradient plasticity J. Mech. Phys. Solids. 49 (2001) 2245-2271.

57. W.D. Nix, H. Gao, Indentation size effects in crystalline materials: a law for strain gradient plasticity, J. Mech. Phys. Solids. 46 (1998) 411-425.

58. M.E. Gurtin, A finite-deformation, gradient theory of single-crystal plasticity with free energy dependent on the accumulation of geometrically necessary dislocations, Int. J. Plast. 26 (2010) 1073-1096.

59. L. Bardella, A deformation theory of strain gradient crystal plasticity that accounts for geometrically necessary dislocationsJ. Mech. Phys. Solids. 54 (2006) 128-160

60. B. Zhu, R.J. Asaro, P. Krysl, R. Bailey, Transition of deformation mechanisms and its connection to grain size distribution in nanocrystalline metals, Acta Mater., 53 (2005), p. 4825

61. B. Zhu, R.J. Asaro, P. Krysl, K. Zhang, J.R. Weertman, Effects of grain size distribution on the mechanical response of nanocrystalline metals: Part II, Acta Materialia, Volume 54, Issue 12, July 2006, Pages 3307-3320

62. R.J. Asaro, P. Krysl, B. Kad, Deformation mechanism transitions in nanoscale fcc metals, Philos Mag Lett, 83 (12) (2003), pp. 733-743

63. Y. Wei, H. Gao, An elastic-viscoplastic model of deformation in nanocrystalline metals based on coupled mechanisms in grain boundaries and grain interiors, Materials Science and Engineering A, 478 (2008), pp. 16-25

64. H. Conrad, J. Narayan, On the grain size softening in nanocrystalline materials, Scr Mater, 42 (2000), pp. 1025-1030

65. D.H. Warner, J.F. Molinari A semi-discrete and non-local crystal plasticity model for nanocrystalline metals, Scripta Materialia, Volume 54, Issue 7, April 2006, Pages 1397-1402

66. E. Gürses, T. El Sayed, A variational multiscale constitutive model for nanocrystalline materials, Journal of the Mechanics and Physics of Solids, 59 (2011), pp. 732-749

67. E. Gürses, T. El Sayed, Constitutive modeling of strain rate effects in nanocrystalline and ultrafine grained polycrystals International Journal of Solids and Structures, Volume 48, Issue 10, 15 May 2011, Pages 1610-1616

68. Y. Aoyagi, T. Tsuru, T. Shimokawa Crystal plasticity modeling and simulation considering the behavior of the dislocation source of ultrafine-grained metal International Journal of Plasticity, Volume 55, April 2014, Pages 43-57

69. J. Shi, M.A. Zikry Grain size, grain boundary sliding, and grain boundary interaction effects on nanocrystalline behaviour, Materials Science and Engineering: A, Volume 520, Issues 12, 15 September 2009, Pages 121-133

70. L. Li,P M. Anderson, MG Lee, E Bitzek, P Derlet, H Van Swygenhoven, The stress-strain response of nanocrystalline metals: A quantized crystal plasticity approach, Acta Materialia, Volume 57, Issue 3, February 2009, Pages 812-822

71. J. Segurado, J. Llorca, Simulation of the deformation of polycrystalline nanostructured Ti by computational homogenization, Comp. Mater. Sci. 76 (2013) 3-11.

72. R. Raj, M.F. Ashby, On grain boundary sliding and diffusional creep, Metall. Trans. A. 2 (1971) 1113-1127.

73. M.F Ashby, R.A Verrall, Diffusion-accommodated flow and superplasticity, Acta Metallurgica, Volume 21, Issue 2, February 1973, Pages 149-163

74. Y.G. Zheng et al., Roles of grain boundary and dislocations at different deformation stages of nanocrystalline copper under tension Phys. Lett. A, 373 (2009) 570-574 
75. R. N. Stevens Grain-boundary sliding and diffusion creep in polycrystalline solids Philosophical Magazine, Volume 23, Issue 182, 1971

76. W. R, Cannon, The contribution of grain boundary sliding to axial strain during diffusion creep, Philosophical Magazine, Volume 25, Issue 6, 1972

77. W. R. Cannon, W. D. Nix Models for grain rearrangement resulting from grain boundary sliding Philosophical Magazine, Volume 27, Issue 1, 1973, pages 9-16

78. I.A. Ovid'ko and A.G. Sheinerman, Kinetics of grain boundary sliding and rotational deformation in nanocrystalline materials., Rev.Adv.Mater.Sci. (RAMS)

No 1, Vol. 35, 2013, pages 48-58

79. A. V. Sergueeva, N. A. Mara, N. A. Krasilnikov, R. Z. Valiev \& A. K. Mukherjee, Cooperative grain boundary sliding in nanocrystalline materials, Philosophical Magazine, Volume 86, Issue 36, 2006 pages 5797-5804

80. H. Hahn, und K. A Padmanabhan. (1977) A model for the deformation of nanocrystalline materials, Philosoph. Magazine, B, 76 (4), pp. 559-571

81. H. Hahn, P. Mondal, K.A. Padmanabhan, Plastic deformation of nanocrystalline materials Nanostructured Materials, Volume 9, Issues 1-8, 1997, Pages 603-606

82. W.Yang, H.T. Wang, Mechanics modeling for deformation of nano-grained metals, Journal of the Mechanics and Physics of Solids, Volume 52, Issue 4, April 2004, Pages 875-889

83. A. Jerusalem, N. Kambouchev and R. Radovitzky, A continuum model of nanocrystalline metals under shock loading, 47th IAA/ASME/ASCE/AHS/ASC Structures, Structural Dynamics, and Materials Conference, 1 - 4 May 2006, Newport, Rhode Island A IAA 20062249

84. D.H. Warner, F. Sansoz, J.F. Molinari, Atomistic based continuum investigation of plastic deformation in nanocrystalline copper, Int J Plasticity, 22 (2006), p. 754

85. P. Barai, G J. Weng, Mechanics of very fine-grained nanocrystalline materials with contributions from grain interior, GB zone, and grain-boundary sliding, International Journal of Plasticity, Volume 25, Issue 12, December 2009, Pages 2410-2434

86. T. Watanabe, An Approach to grain-boundary design for strong and ductile polycrystals, Res Mechanica, v. 11, pp. 47-84, 1984;

87. R. Z. Valiev., I. V. Alexandrov., N. A. Enikeev., et al., Towards enhancement of properties of UFG metals and alloys by grain boundary engineering using SPD processing, Reviews on Advanced Materials Science. 2010, 25: 1-10

88. Handtrack D, Sauer C, Kieback B Microstructure and properties of ultrafine-grained and dispersion-strengthened titanium materials for implants, J Materials Science 43 (2008), 671679

89. X. Sauvage, G. Wilde, S.V. Divinski, Z. Horita, R.Z. Valiev, Grain boundaries in ultrafine grained materials processed by severe plastic deformation and related phenomena, Materials Science and Engineering: A, Vol. 540, 1 April 2012, Pages 1-12

90. I. Semenova et al, Enhanced strength and ductility of ultrafine-grained Ti processed by severe plastic deformation, Advanced Engineering Materials, Special Issue: Bulk Nanostructured Materials, Volume 12, Issue 8, pp. 803-807, August, 2010

91. X. Sauvage, et al., Grain boundaries in ultrafine grained materials processed by severe plastic deformation and related phenomena, Materials Science \& Engineering: A, Vol. 540, 12012 , Pp. 1-12

92. A. Hasnaoui, H .V. Swygenhoven, P. M Derlet, On non-equilibrium grain boundaries and their effect on thermal and mechanical behaviour: A Molecular dynamics computer simulations, Acta Mater. 50 (2002) 3927-3939. 
93. G.J. Tucker, D.L. McDowell, Non-equilibrium grain boundary structure and inelastic deformation using atomistic simulations, Int. J. Plast. 27 (2011) 841-857.

94. A Hasnaoui, H Van Swygenhoven, P.M DerletOn non-equilibrium grain boundaries and their effect on thermal and mechanical behaviour: a molecular dynamics computer simulation, Acta Materialia, Volume 50, Issue 15, 3 September 2002, Pages 3927-3939

95. V.N. Chuvil'deev, Non-equilibrium grain boundary in metals, Theory and applications, Fizmatlit, Moscow, 2004.

96. V.N. Chuvil'deev, Theory of non-equilibrium grain boundaries and its applications for nanoand microcrystalline materials processed by ECAP. Annales de Chimie-Science des Materiaux (2002)

97. H.S. Liu, W. Pantleon, L.Mishnaevsky Jr., , Non-equilibrium grain boundaries in UFG titanium: Computational study of sources of the material strengthening, Comp. Mater. Sci. 83 (2014) 318-330.

98. V. Péron-Lührs, A. Jérusalem, F. Sansoz, L. Stainier, L. Noels, A two-scale model predicting the mechanical behavior of nanocrystalline solids, Journal of the Mechanics and Physics of Solids, Volume 61, Issue 9, September 2013, Pages 1895-1914

99. R. Dobosz, M. Lewandowska, K. J. Kurzydlowski FEM modelling of the combined effect of grain boundaries and second phase particles on the flow stress of nanocrystalline metals, Computational Materials Science, Volume 53, Issue 1, February 2012, Pages 286-293

100. K.S. Kumar et al. Deformation of electrodeposited nanocrystalline nickel Acta Mater 2003;51:387

101. K.S Kumar, H. Van Swygenhoven, S Suresh, Mechanical behavior of nanocrystalline metals and alloys, Acta Materialia, Volume 51, Issue 19, 25 2003, Pages 5743-5774

102. J. Cizek et al, Ultra fine-grained metals prepared by severe plastic deformation: A positron annihilation study Acta Physica Polonica A 107 , 745 (2005)

103. A. Hohenwarter, R. Pippan, A comprehensive study on the damage tolerance of ultrafinegrained copper, Materials Science and Engineering: A, Volume 540, 1 April 2012, Pages 89-96

104. Chuvil'deev, V.N. Non-equilibrium grain boundary in metals. Theory and applications, Fizmatlit, Moscow, 2004;

105. Wei YJ, Anand L. Grain-boundary sliding and separation in polycrystalline metals: application to nanocrystalline fcc metalsJ Mech Phys Solids 2004;52:2587.

106. Y J Wei, C. Su, L. Anand A computational study of the mechanical behavior of nanocrystalline fcc metals, , Acta Materialia 54 (2006) 3177-3190

107. L Chen and YG Wei Overall mechanical behavior of nanocrystalline materials accompanied by damage evolution on grain boundaries nternational Journal of Damage Mechanics, 2014, Vol 23(1) 25-42

108. P. Gu, M.Dao, R. J. Asaro, S. Suresh, A unified mechanistic model for size-dependent deformation in nanocrystalline and nanotwinned metals, Acta Materialia, Volume 59, Issue 18, October 2011, Pages 6861-6868

109. A. Siddiq, T. El Sayed A phenomenological variational multiscale constitutive model for intergranular failure in nanocrystalline materials, Materials Letters, Volume 107, 15 September 2013, Pages 56-59

110. L. Mishnaevsky Jr and P. Brøndsted, Three-dimensional numerical modelling of damage initiation in UD fiber-reinforced composites with ductile matrix, Materials Science \&Engineering: A, Vol.498, No. 1-2, 2008, pp. 81-86 
111. L. Mishnaevsky Jr, Lippmann N; Schmauder S, Computational modeling of crack propagation in real microstructures of steels and virtual testing of artificially designed materials , Int J Fracture, Vol. 120 No. 4 pp. 581-600, 2003

112. J. Lin, Y.Liu, T.A. Dean, A Review on Damage Mechanisms, Models and Calibration Methods under Various Deformation Conditions, Int. J Damage Mechanics, 2005 vol. 14 no. 4 299-319

113. Lin, J., Hayhurst, D.R. and Dyson, B.F. (1993). The Standard Ridges Uniaxial Testpiece: Computed Accuracy of Creep Strain , J. of Strain Analysis, 28(2): 101-115

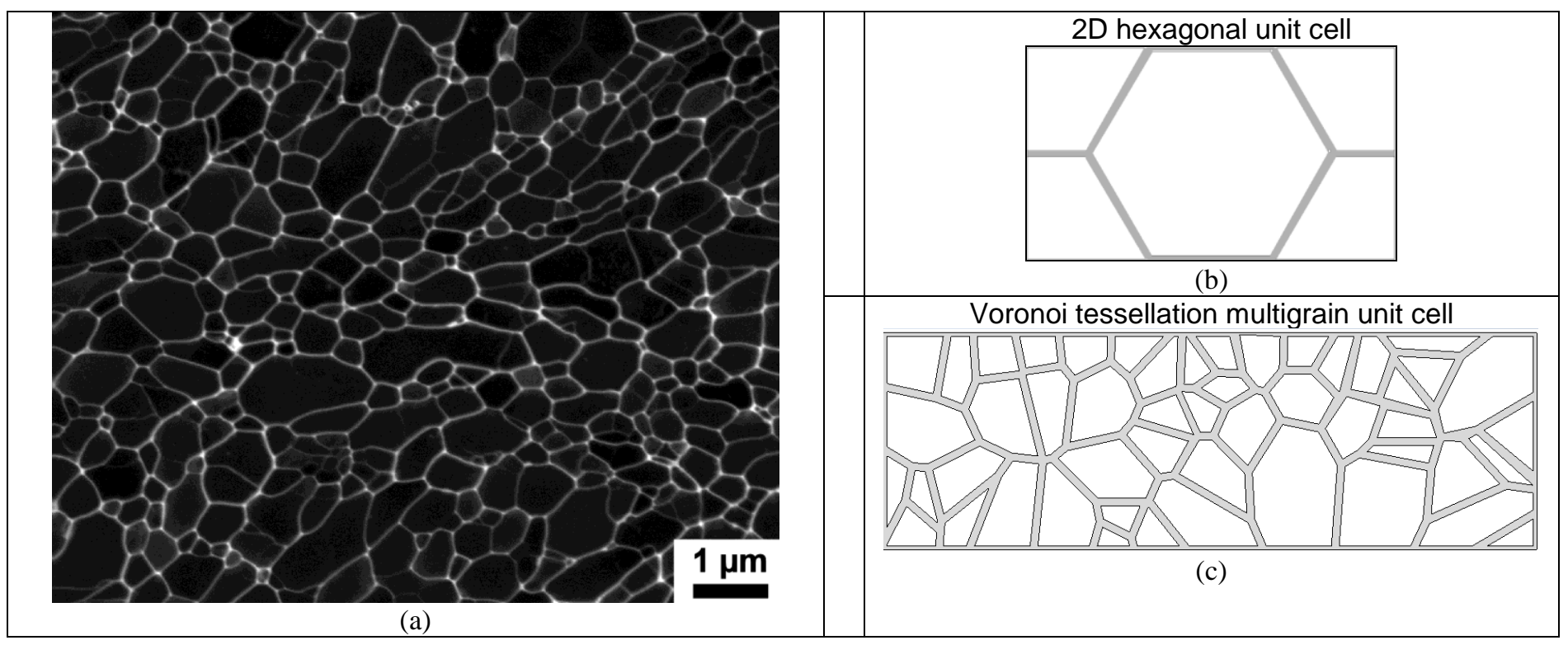

Figure 1. Real microstructure and unit cell models: (a) Microstructure of heavily deformed commercial purity aluminum [9] revealed using gallium enhanced microscopy [10] (courtesy of O.V.Mishin). (b) Hexagonal unit cell idealization, with finite thickness grain boundaries, (c) Voronoi tessellation unit cell, with finite thicjness grain boundaries $[26,88]$

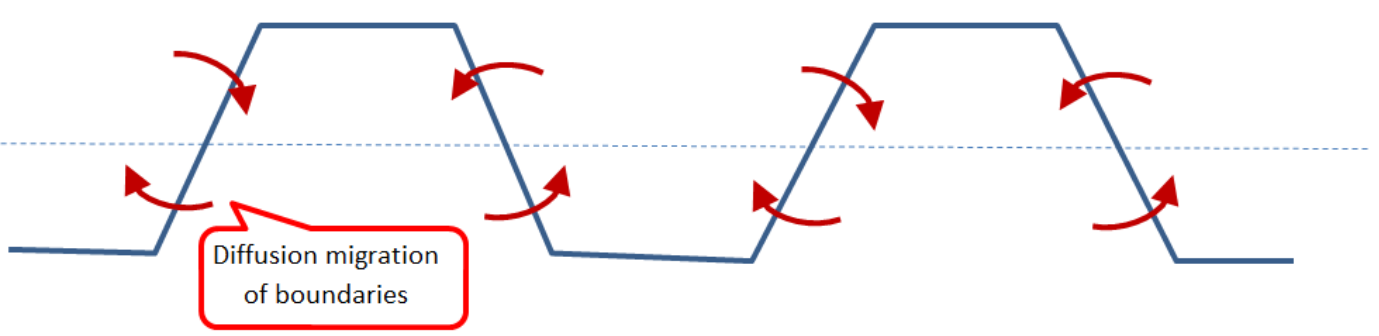

Figure 2. A model of GBS path according to Fu et al. [14] 


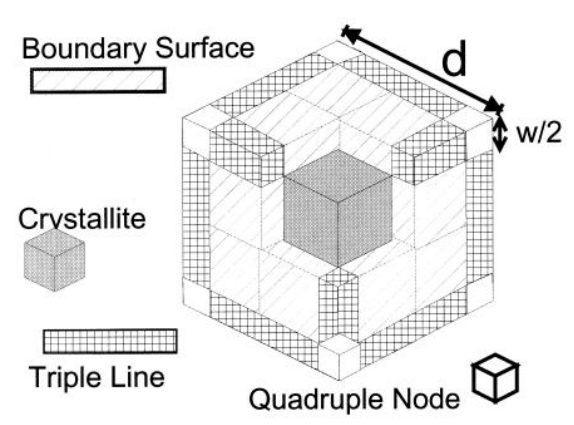

(a)
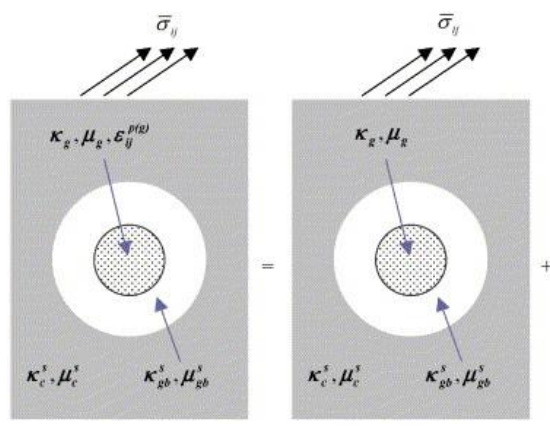

(b)

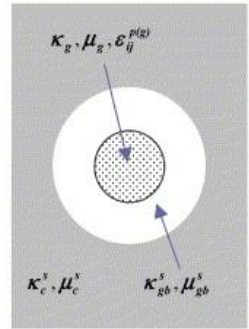

Figure

3. Examples of unit cell models of nanocrystalline materials: Cubic unit cell model by Kim [17](a) and self-consistent polycrystal model by Jiang and Weng [33] presented as superposition of two linear problems. Reprinted with kind permission from Elsevier.

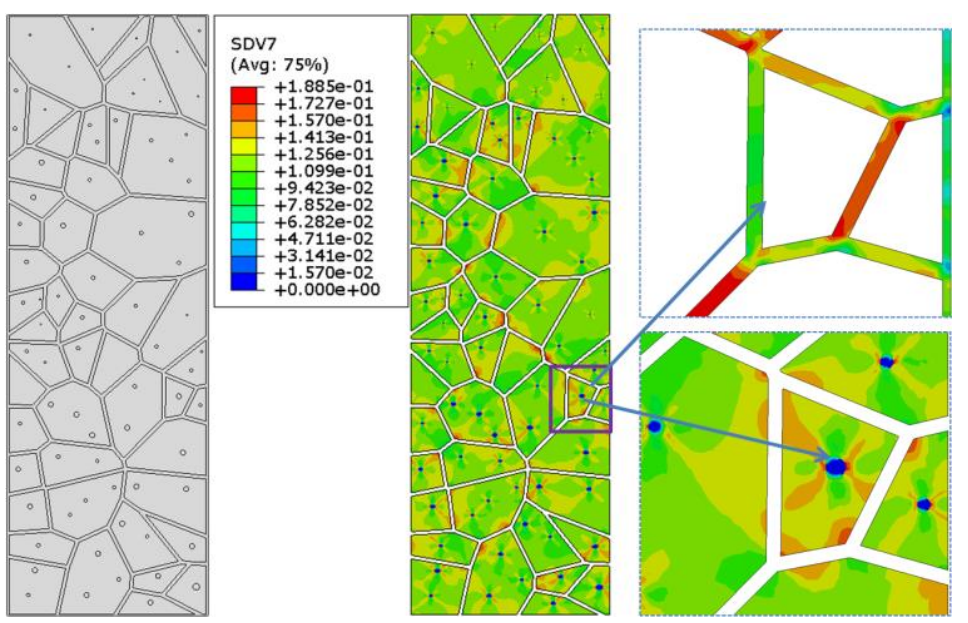

(a)
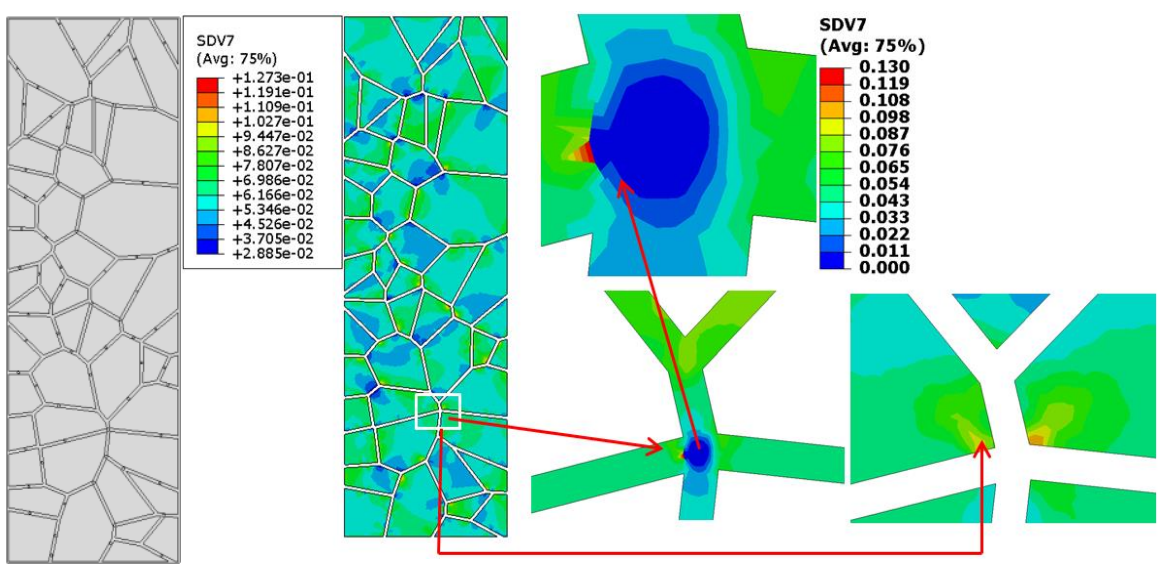

(b) 
Figure 4. Precipitates in grain interior (A) and GB (B) of UFG titanium: Computational study of the precipitate effect on damage distribution. Reprinted from [26] with kind permission from Elsevier. 